\title{
Anabases
}

ANABASES Traditions et réceptions de l'Antiquité

25 | 2017

Varia

\section{Luciano CANFORA, Il presente come storia. Perché il passato ci chiarische le idee}

\section{Márcio Suzuki}

\section{OpenEdition}

\section{Journals}

Edizione digitale

URL: http://journals.openedition.org/anabases/6120

DOI: 10.4000/anabases.6120

ISSN: 2256-9421

\section{Editore}

E.R.A.S.M.E.

\section{Edizione cartacea}

Data di pubblicazione: 1 avril 2017

Paginazione: 278-280

ISSN: 1774-4296

Notizia bibliografica digitale

Márcio Suzuki, «Luciano canfora, Il presente come storia. Perché il passato ci chiarische le idee»,

Anabases [Online], 25 | 2017, Messo online il 01 avril 2017, consultato il 19 janvier 2021. URL: http:// journals.openedition.org/anabases/6120 ; DOI: https://doi.org/10.4000/anabases.6120

Questo documento è stato generato automaticamente il 19 janvier 2021.

(c) Anabases 


\title{
Luciano CANFORA, Il presente come storia. Perché il passato ci chiarische le idee
}

\author{
Márcio Suzuki
}

\section{NOTIZIA}

Luciano CANFORA, Il presente come storia. Perché il passato ci chiarische le idee

Milano, Rizzoli, 2014, 265 p.,

10 euros/ ISBN 978-88-17-07572-5

1 I tempi attuali sono stati eloquenti nello smentire la tesi della fine della storia. Il libro recente di Luciano Canfora - Il presente come storia - non ha lo scopo di rafforzare questa evidenza, ma di mostrare, contro ogni tentativo politico-intellettuale di fare tacere la storia, che il presente è quasi sempre una attualità del passato. Gli articoli che compongono il volume sono brevi, di lettura agevole e contundenti, come delle frecce corte e letali, confezionate da una vasta erudizione e da una vigorosa passione politica. Sarebbe interessante investigare i modelli di questa eloquenza che collega conoscenza storica e politica. Ad ogni modo, è certo che l'autore è mosso dalla stessa "morale profonda" che conduce lo scrittore Ivan Jablonka a investigare la scomparsa della propria famiglia ad Auschwitz - morale profonda e salutare per ogni investigazione storica : afferma Jablonka che "non ha senso contrapporre scientificità e partecipazione emotiva, eventi esterni e passione di chi li comunica, storia e arte del racconto, perché l'emozione non nasce dal pathos o dall'accumulo di superlativi : essa scaturisce dalla nostra tensione verso la verità".

2 La lettura degli articoli isolati (molti sono recensioni) non stona nella resa dell'insieme, perché a legarli vi è un dispositivo comune : gli episodi passati e le loro versioni hanno il potere di illuminare la politica attuale. La semplicità pedagogica del sottotitolo "perché il passato ci chiarisce le idee" - si ridurrebbe ad una trivialità senza il 
momento effettivamente rivelatore, la capacità di scoprire nel passato la trama che smaschera la supposta neutralità, il cinismo, la falsificazione del presente; smascheramento che non può essere portato a termine senza una ricostruzione minuziosa dell'evento remoto che serve da punto di partenza per l'interpretazione del momento attuale. La chiave di interpretazione è nell'osservazione di certe ricorrenze storiche. Un esempio tra tanti, ma di cogente attualità, è quello della guerra tra Roma $\mathrm{e}$ i Cartaginesi : "L'invenzione del nemico" - scrive Canfora - "nel senso della capacità di imporne un'immagine demonizzante [...] è una delle armi più importanti nei conflitti di potenza". Nel caso della costruzione del nemico cartaginese, la lezione non propizia soltanto una illazione generale, ma è accompagnata da una triste ripetizione dell'avvenuto quasi esattamente nello stesso luogo : "l'ironia della storia ha voluto che in quest'ultimo tempo della tormentata storia del Nordafrica la vicenda si ripetesse, negli stessi luoghi, o quasi, in cui avvenne l'antico sterminio: il rapace Sarkozy che 'libera' la Libia rassomiglia, in caricatura, a Scipione Emiliano, detto Africano minore, che assedia e distrugge Cartagine nel nome della 'libertà' e attraverso una 'guerra giusta". Non mancano esempi della ferocità dei vincitori, e Simone Weil, ricorda l'autore, già richiamava l'attenzione sulle somiglianze tra lo sterminio dei Galli da parte dei Romani e quella degli Ebrei sotto il nazismo.

Il problema dell'invenzione del nemico è che essa presuppone la sua distruzione. La sua demonizzazione ne implica necessariamente l'annichilamento, ciò che forse vale tanto per la guerra come per la politica. Questa invenzione avrebbe conseguenze in due ambiti diversi. Sul piano esterno, la "ferocia dei vincitori" si fa vedere nella voracità con cui si spogliano i vinti, e ci si mantiene al potere mediante la costruzione di nuovi nemici, con l'alimentazione costante della macchina bellica. Sul piano interno, la ferocia s'annida nella fallacia implicita al sistema democratico : chi vince le elezioni oggi, per più piccola che sia la differenza dei voti e per più ampia che sia l'astensione dal suffragio, si crede nel diritto di credere che il bottino gli appartiene completamente. Ciò aumenta soltanto la crisi di legittimità e di rappresentatività. La fallacia della maggioranza sarebbe stata constatata tra gli antichi, che non dubitavano, con elementi pertinenti, della procedura con cui si decide attraverso la maggioranza. Tra di essi i due grandi filosofi dell'antichità. Da una parte, Aristotele, nel rifiutare la "legge del numero" quando osserva, nella Politica, che "la democrazia non è il governo della maggioranza, ma il governo dei poveri", essendo questi in genere la maggioranza. D'altra parte, Platone, nel prestare la voce alla tipica critica alla democrazia quando parla in nome della competenza, che può trasformarsi in "un altro modo per dire ricchezza". Questa sarebbe - prosegue l'autore - "l'architrave della critica liberale alla democrazia" durante tutto l'Ottocento. Ancora peggio : "in tempi ancor a noi più vicini la prevalenza del pensiero democratico su quello liberale, affermatosi, ad esempio, nelle codificazioni 'costituzionali' del secondo dopoguerra, è venuta declinando, e ha ceduto il passo al ritorno in grande stile del predominio dei 'competenti' : o di coloro che, intrinseci al mondo arduo delle finanze, si pretendono tali". Il rito elettorale passa ad essere soltanto il fattore di legittimazione del potere realmente in mano a tecnici di supposta competenza, raramente eletti nelle urne.

4 Lungi da qualsiasi smascheramento vuoto, da un semplice decostruzionismo che fa tabula rasa della storia, la vera demitificazione richiede l'attualità della storia. È possibile trovare delle ripetizioni che conducono a percepire una certa reiterazione, una continuità che soggiace al corso storico e permette di rendere conto di un largo movimento. Un chiaro esempio in questa direzione è la discussione sul senso della 
maltrattata parola "democrazia", usata per squalificare gli Stati che non ne seguono i principi, ma che vede diminuita l'ampiezza della sua portata quanto utilizzata all'interno delle proprie mura. Illuminante la ripresa della democrazia romana in detrimento di quella ateniese dal pensiero politico anglosassone moderno: le ragioni che portano a rifiutare quest'ultima sono dovute al fatto che la costituzione romana offre il modello del sistema misto, in cui il potere del popolo è limitato e controllato dal senato.

5 Le mani abili che tessono i fili del libro lasciano poco margine all'ottimismo : i momenti in cui la storia può essere dipinta con colori più allegri sono rarissimi. La sobrietà con cui si devono esaminare le reiterazioni storiche è l'antidoto migliore al pensiero determinista che vuole spiegare, prevedere e anche imporre i prossimi passi della storia, o alla concezione pacificatrice che dice che la storia è approdata al suo fine. L'idea che il socialismo sia l'ultima tappa dello sviluppo umano presenta, perciò, una forte affinità con l'illusione liberale a riguardo della "eternità" del capitalismo : "Una delle illusioni ricorrenti del pensiero umano è di ritenere di vivere il punto di arrivo della storia. Non è esatto che tale veduta sia stata caratteristica soltanto del pensiero antico, privo di mentalità storicistica". Guardare al passato sarebbe una forma di non avere illusione con il futuro? Se fosse così, sarebbe interessante sapere quello che pensa l'autore delle tesi sull'imminenza della fine del capitalismo o di quelle che parlano di un'era post capitalistica.

6 La storia come disciplina non si oppone alla storia come emozione. La composizione adeguata di entrambe permette allo storico di posizionarsi ad uguale distanza dall'adesione ingenua al passato o al futuro, e dalla freddezza o indifferenza in relazione al presente. L'indignazione con lo status quo, con i tentativi di rendergli un'aria di normalità è ciò che motiva la ricerca storica, allora non più certamente asettica, perché bagnata in un distanziamento interessato: sono magistrali le pagine che oppongono l'aderenza di Machiavelli all'antichità (il plagio come omaggio) e il lavoro di liberazione in relazione agli archetipi antichi da parte di Guicciardini. In Machiavelli si scorge il cuore dello storico: il nuovo pensiero nasce dal dialogo, dalla riflessione sugli antichi, non trattandosi di un "culto subalterno del passato o di una soggezione classicizzante", bensì della convinzione che nel passato remoto vi è un "accumulo di esperienza e pensiero che è ancora in attesa di essere sfruttato fino in fondo" ("io mi trasferisco completamente in essi", scrive lui a Vettori). Invece la "assimilazionesuperamento" in Guicciardini conosce un distanziamento : anticipando ciò che Borges dirà sullo stile di Gibbon in chiave positiva, Guicciardini percepisce che l'identificazione tra presente e passato è solo possibile grazie alla stilizzazione, un altro nome per l'ignoranza dei dettagli. La "lente deformante" che permette la proiezione del passato nel presente si deve alla carenza di elementi materiali, insomma, alla mancanza di una ricerca più attenta.

7 Se la storia insegna che non vi è una teleologia, una filosofia della storia, questo non vuol dire che la storia non sia profondamente filosofica, che non abbia la sua "morale profonda". L'idea di una ricorrenza dell'evento, di una ripresa del passato come critica alla sua identificazione mitico-ideologica, può ricordare la "teoria della storia" e le critiche politiche di Heinrich Heine, scritte al caldo delle rivoluzioni francesi del $1830 \mathrm{e}$ del 1848 - Heine, da chi Marx ha preso in prestito l'idea che la storia si ripete, la prima volta come tragedia, la seconda come farsa. Con l'incisività di questi ammirevoli scrittori, osservatori acuti dei loro tempi, le pagine di questo libro vanno un po' oltre : 
se si sa bene osservare, ciò che si vede non è tanto la messinscena della farsa, ma la ripetizione del disastro.

\section{AUTORI}

MÁRCIO SUZUKI

Università di San Paolo, Brasile

marciosuzuki@usp.br 